\title{
Spherical Gravitational Collapse of Anisotropic Radiating Fluid Sphere
}

\author{
B. C. Tewari1, Kali Charan², Jyoti Rani1 \\ ${ }^{1}$ Department of Mathematics, Kumaun University, SSJ Campus, Almora, India \\ ${ }^{2}$ Department of Mathematics, SRMS College of Engineering and Technology, Bareilly, India \\ Email:drbctewari@yahoo.co.in, kcyadav2008@gmail.com
}

Received 22 March 2016; accepted 15 May 2016; published 18 May 2016

Copyright $(2016$ by authors and Scientific Research Publishing Inc.

This work is licensed under the Creative Commons Attribution International License (CC BY).

http://creativecommons.org/licenses/by/4.0/

c) (i) Open Access

\begin{abstract}
We here present a relativistic model for a spherically symmetric anisotropic fluid to study the various factors of physical and thermal phenomenon during the evolution of a collapsing star dissipating energy in the form of radial heat flow. We also proposed a table of some new parametric class of solutions which will be useful for constructing the new compact star models. The constructed algorithm obeys all the relevant requirements of a realistic model and matched with Vaidya exterior metric over the boundary. At the initial stage the interior solutions represent a static configuration of perfect fluid which then gradually starts evolving into radiating collapse. The apparent luminosity as observed by the distant observer at rest at infinity and the effective surface temperature are zero in remote past at the instant when collapse begins and at the stage when collapsing configuration reaches the horizon of the black hole.
\end{abstract}

\section{Keywords}

Exact Solutions, Anisotropic Fluid, Spherical Gravitational Collapse, Black Hole

\section{Introduction}

In modern Astrophysics and Cosmology, a detailed description of gravitational collapse of massive stars and the modeling of the structure of compact objects such as Neutron star, Quasar, Supernovae, Black hole etc. under various conditions is the most interesting phenomena. The final outcome of the gravitational collapse is an important open issue in relativistic astrophysics (Joshi and Malafarina [1] and references therein). There is no iron-clad evidence that black hole candidates are indeed black holes. There is no logic that prevents existence of naked singularities and as per Cosmic Censorship Conjecture Penrose [2]; himself considers this an open question. Understanding the characteristics and features of final fate of a collapsing system is important from 
both the perspectives theoretical as well as observational (Virbhadra [3] and references therein).

In order to construct the new realistic models, it is desirable to solve the Einstein's field equations but due to non linear character of the equations it is a very difficult task; various efforts have been made in this direction. The maiden exact solution of spherical gravitational collapse was due to Oppenheimer and Snyder [4] and which apparently suggested that such a collapse results in formation of Black Holes. Taking into account the outgoing radiation from collapsing spherical fluid Vaidya [5] [6] initiated the problem describing the exterior field and Santos [7] presented the junction conditions of collapsing spherically symmetric shear-free non-adiabatic fluid with radial heat flow.

Herrera and Santos [8] and Mitra [9] established the fact that gravitational collapse is a high energy dissipating process which plays a dominant role in the formation and evolution of stars. Historically the dissipation of energy from collapsing fluid distribution is described in two limiting cases. The first case describes the free streaming approximation (pure radiation) while second one is diffusion approximation (heat flow). The prominent work in pure radiation is due to Tewari and he solved the Einstein's field equations with a new approach and developed the Quasar models [10]-[13]. While a number of realistic models in diffusion approximation with the impact of various factors such as inhomogeneity, anisotropy, viscosity, electromagnetic field and various dissipative processes on the evolution are critically discussed by de Oliveira et al. [14]; Bonnor et al. [15]; Herrera et al. [16]; Maharaj and Govender [17]; Ivanov [18]; Tewari [19] [20]; Pinheiro and Chan [21]; Tewari and Charan [22] [23]; Sharif and Iftikhar [24].

Bowers and Liang [25] did the pioneering work in the field of anisotropic fluid models which enabled researchers to study the effect of anisotropy on the physical behavior of a star undergoing gravitational collapse. Herrera and Santos [26] explored the properties of anisotropic self-gravitating spheres using the perturbation method. Herrera et al. [27] investigated that the local pressure anisotropy is one of the responsible factors for inhomogeneities in energy density and in this series some interesting noticeable work is reported by Ivanov [28] [29]; Reddy et al. [30]; Tewari and Charan [31] [32]; Govender et al. [33] and a different approach with a new concept Horizon function is very recently introduced for anisotropic fluids by Ivanov [34] [35].

The main objective of this work is to present a simple anisotropic collapsing radiating fluid model and discuss all the relevant thermal and physical conditions by taking Tewari and Charan [31] solution as seed solution and we propose some new exact solutions for this parametric class of solution which are useful in construction of the compact stars models. The interior metric is spherically symmetric, shear-free, anisotropic radiating away its energy in the form of radial heat and contracting in size during the process of collapse. The interior metric matched with the Vaidya exterior metric [6] over the boundary. Final fate of our model is the formation of a black hole. The paper is organised as: In Section 2 the space-times and the junction conditions for collapsing system are given. In Section 3 solution of Einstein's field equations is presented. Section 4 describes a parametric class of solution and some new solutions. In Section 5 detailed study of the model in which we have obtained expressions of various physical parameters is described. Section 6 contains the temperature profile of the collapsing body. Finally in Section 7 some concluding remarks have been made.

\section{The Space-Times and Junction Conditions}

The interior space-time of a shear-free spherically symmetric fluid with the coordinates $(t, r, \theta, \phi)$ is given by

$$
\mathrm{d} s_{-}^{2}=-A^{2}(r, t) \mathrm{d} t^{2}+B^{2}(r, t)\left\{\mathrm{d} r^{2}+r^{2}\left(\mathrm{~d} \theta^{2}+\sin ^{2} \theta \mathrm{d} \phi^{2}\right)\right\}
$$

The energy-momentum tensor for the matter distribution with anisotropy in pressure is

$$
T_{\mu \nu}=\left(\epsilon+p_{t}\right) w_{\mu} w_{v}+p_{t} g_{\mu v}+\left(p_{r}-p_{t}\right) x_{\mu} x_{v}+q_{\mu} w_{v}+q_{v} w_{\mu}
$$

where $\epsilon$ is the energy density of the fluid, $p_{r}$ the radial pressure, $p_{t}$ the tangential pressure, $w_{\mu}$ is the fourvelocity, $q_{\mu}$ the radial heat flow vector and $x_{\mu}$ is a unit space like four vector along the radial direction.

Assuming comoving coordinates, we have $w^{\mu}=\frac{1}{A} \delta_{0}^{\mu}$. The heat flow vector $q^{\mu}$ is orthogonal to the velocity vector so that $q^{\mu} w_{\mu}=0$ and takes the form $q^{\mu}=q \delta_{1}^{\mu}$.

Since the interior fluid is radiating energy in the form of heat therefore the exterior space-time of a collapsing radiating star is described by Vaidya's outgoing exterior metric [6] 


$$
\mathrm{d} s_{+}^{2}=-\left(1-\frac{2 M(v)}{R}\right) \mathrm{d} v^{2}-2 \mathrm{~d} R \mathrm{~d} v+R^{2}\left(\mathrm{~d} \theta^{2}+\sin ^{2} \theta \mathrm{d} \phi^{2}\right)
$$

where $v$ is the retarded time and $M(v)$ is the Vaidya mass.

The junction conditions for radiating star matching two line elements (1) and (3) at the boundary continuously across a spherically symmetric time-like hyper surface $\Sigma$ are very well known and given by Santos [7]

$$
\begin{gathered}
(r B)_{\Sigma}=R_{\Sigma}(v) \\
\left(p_{r}\right)_{\Sigma}=(q B)_{\Sigma} \\
m_{\Sigma}(r, t)=M(v)=\left\{\frac{r^{3} B \dot{B}^{2}}{2 A^{2}}-r^{2} B^{\prime}-\frac{r^{3} B^{\prime 2}}{2 B}\right\}_{\Sigma}
\end{gathered}
$$

where $m_{\Sigma}$ is the mass function calculated in the interior at $r=r_{\Sigma}$ (Cahill et al. [36]; Misner and Sharp [37]). The primes and dots stand for differentiation with respect to $r$ and $t$ respectively.

Some other physical and thermal features of the collapsing matter are the surface luminosity and the boundary redshift $z_{\Sigma}$ observed on $\Sigma$ are

$$
\begin{gathered}
L_{\Sigma}=\frac{\kappa}{2}\left\{r^{2} B^{3} q\right\}_{\Sigma} \\
Z_{\Sigma}=\left[1+\frac{r B^{\prime}}{B}+\frac{r \dot{B}}{A}\right]_{\Sigma}^{-1}-1
\end{gathered}
$$

The total luminosity for an observer at rest at infinity is

$$
L_{\infty}=-\frac{\mathrm{d} M}{\mathrm{~d} v}=\frac{L_{\Sigma}}{\left(1+z_{\Sigma}\right)^{2}}
$$

\section{Solution of Einstein's Field Equations}

In order to solve the Non-trivial Einstein's field equations which are generated by (1) and (2), we choose a separable form of the metric coefficients given in (1) into functions of $r$ and $t$ coordinates as $A(r, t)=A_{0}(r) g(t)$ and $B(r, t)=B_{0}(r) f(t)$. The coupling constant in geometrized units is taken as $\kappa=8 \pi(i . e . G=c=1)$ and in view of (1) and (2) with the help of separable metric coefficients we get the following expressions for field equations

$$
\begin{gathered}
\kappa \epsilon=\frac{\epsilon_{0}}{f^{2}}+\frac{3 \dot{f}^{2}}{A_{0}^{2} g^{2} f^{2}} \\
\kappa p_{r}=\frac{\left(p_{r}\right)_{0}}{f^{2}}+\frac{1}{A_{0}^{2} g^{2}}\left(-\frac{2 \ddot{f}}{f}-\frac{\dot{f}^{2}}{f^{2}}+\frac{2 \dot{f} \dot{g}}{f g}\right) \\
\kappa p_{t}=\frac{\left(p_{t}\right)_{0}}{f^{2}}+\frac{1}{A_{0}^{2} g^{2}}\left(-\frac{2 \ddot{f}}{f}-\frac{\dot{f}^{2}}{f^{2}}+\frac{2 \dot{f} \dot{g}}{f g}\right) \\
\kappa q=-\frac{2 A_{0}^{\prime} \dot{f}}{A_{0}^{2} B_{0}^{2} g f^{3}}
\end{gathered}
$$

where

$$
\epsilon_{0}=-\frac{1}{B_{0}^{2}}\left(\frac{2 B_{0}^{\prime \prime}}{B_{0}}-\frac{B_{0}^{\prime 2}}{B_{0}^{2}}+\frac{4 B_{0}^{\prime}}{r B_{0}}\right)
$$




$$
\begin{aligned}
& \left(p_{r}\right)_{0}=\frac{1}{B_{0}^{2}}\left(\frac{B_{0}^{\prime 2}}{B_{0}^{2}}+\frac{2 B_{0}^{\prime}}{r B_{0}}+\frac{2 A_{0}^{\prime} B_{0}^{\prime}}{A_{0} B_{0}}+\frac{2 A_{0}^{\prime}}{r A_{0}}\right) \\
& \left(p_{t}\right)_{0}=\frac{1}{B_{0}^{2}}\left(\frac{B_{0}^{\prime \prime}}{B_{0}}-\frac{B_{0}^{\prime 2}}{B_{0}^{2}}+\frac{B_{0}^{\prime}}{r B_{0}}+\frac{A_{0}^{\prime \prime}}{A_{0}}+\frac{A_{0}^{\prime}}{r A_{0}}\right)
\end{aligned}
$$

Here the quantities with the suffix 0 corresponds to the static star model with metric components $A_{0}(r), B_{0}(r)$.

In the absence of non-adiabatic dissipative forces the Equation (5), $\left(p_{r}\right)_{\Sigma}=(q B)_{\Sigma}$, reduces to the condition $\left[\left(p_{r}\right)_{0}\right]_{\Sigma}=0$ and yields at $r=r_{\Sigma}=R_{\Sigma}$

$$
\frac{2 \ddot{f}}{f}+\frac{\dot{f}^{2}}{f^{2}}-\frac{2 \dot{g} \dot{f}}{g f}=\frac{2 \alpha g \dot{f}}{f^{2}}
$$

where

$$
\alpha=\left(\frac{A_{0}^{\prime}}{B_{0}}\right)_{\Sigma}
$$

Tewari [20] by assuming $g(t)=f(t)$ solved the Equation (17) and the solution so obtained is identical to the solution presented by de Oliveira et al. [14] and Bonnor et al. [15] with $g(t)=1$.

$$
\begin{gathered}
\dot{f}=-2 \alpha \sqrt{f}(1-\sqrt{f}) \\
t=\frac{1}{\alpha} \ln (1-\sqrt{f})
\end{gathered}
$$

Here we observed that the function $f(t)$ decreases monotonically from the value $f(t)=1$ at $t=-\infty$ to $f(t)=0$ at $t=0$. It interpret that the collapse begins in the remote past and gradually starts evolve into radiating collapse.

\section{The New Parametric Class of Solution and Some Proposed Solutions}

To find a new parametric class of exact solutions of pressure anisotropy equation which is created by the Equations (11) and (12), Tewari and Charan [31] assumed that the anisotropy evolves

$\kappa\left(p_{t}-p_{r}\right)=\Delta(r, t)=\frac{\delta(r)}{B_{0}^{2}(r) f^{2}(t)}$ and got the following time independent differential equation

$$
\frac{A_{0}^{\prime \prime}}{A_{0}}+\frac{B_{0}^{\prime \prime}}{B_{0}}-\frac{A_{0}^{\prime}}{r A_{0}}-\frac{B_{0}^{\prime}}{r B_{0}}-\frac{2 B_{0}^{\prime 2}}{B_{0}^{2}}-\frac{2 A_{0}^{\prime} B_{0}^{\prime}}{A_{0} B_{0}}-\delta(r)=0
$$

Making an adhoc relationship between the variables in (21), the above mentioned authors obtained the following solution

$$
\begin{gathered}
A_{0}=D_{2}\left(1+D_{1} r^{2}\right)\left(1+C_{1} r^{2}\right)^{\frac{n}{1+1}} \\
B_{0}=C_{2}\left(1+C_{1} r^{2}\right)^{\frac{1}{1+1}} \\
\delta(r)=\frac{(2 n-2)}{(l+1)} \frac{4 D_{1} C_{1} r^{2}}{\left(1+D_{1} r^{2}\right)\left(1+C_{1} r^{2}\right)}
\end{gathered}
$$

where $n, l, C_{1}, C_{2}, D_{1}$ and $D_{2}$ are constants and 


$$
n=\frac{1}{2}\left\{(l+3) \pm\left(l^{2}+10 l+17\right)^{\frac{1}{2}}\right\}
$$

where $n$ is real if $l \geq-5+2 \sqrt{2}$ or $l \leq-5-2 \sqrt{2}$.

For different values of $n$ or $l$ Equations (22) and (23) give a variety of solutions and they are categorized as isotropic pressure and homogeneous density, isotropic pressure and inhomogeneous density while some inhomogeneous density and anisotropic pressure. For $n=0$ we get the homogeneous density and anisotropic pressure, for $n=-1$ we get the isotropic pressure and homogeneous density and in this case solution reduces to Banerjee et al. [38] and for $n=1$ we get isotropic pressure and inhomogeneous density. In order to maintain the inhomogeniety and anisotropy for a collapsing radiating star, a Horizon-free case has been studied by Tewari and Charan [31] for $n=-1-\sqrt{2}$ and in an other study the same authors presented a Black hole model for $n=-2$ [32]. Since a number of solutions can be obtained with the above mentioned parametric class of solution, so keeping this point in mind we here find some exact solutions and listed them in a table and they will be fruitful for further study to construct the various models of radiating and static stars.

\section{Physical and Thermal Analysis of a Specific Model}

To construct a new relativistic model, in the present study we assume $n=0$, to maintain the anisotropy for a collapsing radiating star and using (22)-(24) we get

$$
\begin{gathered}
A_{0}=D_{2}\left(1+D_{1} r^{2}\right) \\
B_{0}=C_{2}\left(1+C_{1} r^{2}\right)^{-1} \\
\delta(r)=\frac{8 D_{1} C_{1} r^{2}}{\left(1+D_{1} r^{2}\right)\left(1+C_{1} r^{2}\right)} \\
\left(p_{r}\right)_{0}=\frac{4 C_{1}}{C_{2}^{2}}\left[\frac{D_{1}\left(1-C_{1}^{2} r^{4}\right)}{C_{1}\left(1+D_{1} r^{2}\right)}-1\right] \\
\left(p_{t}\right)_{0}=\frac{12 C_{1}}{C_{2}^{2}}\left[\frac{C_{1}^{2}}{D_{1}\left(1+C_{1} r^{2}\right)^{2}}-1\right]
\end{gathered}
$$

The junction condition $\left\{\left(p_{r}\right)_{0}\right\}_{\Sigma}=0$ gives

$$
D_{1}=\frac{C_{1}}{1-C_{1} r_{\Sigma}^{2}-C_{1}^{2} r_{\Sigma}^{4}}
$$

Here from (30) and (31), we are seeing that at the centre radial and transverse pressures are equal and anisotropy vanishes there.

A physically reasonable solution should satisfy certain energy conditions and they are:

The central values of both the pressures, energy density and gravitational potential should be non-zero positive definite and the solution should have monotonically decreasing expressions for the pressures and density with the increase of $r$. Thus in view of these conditions, we write the bounds of model parameters $C_{1}>0, C_{2}>0, D_{2}>0$ and $2 C_{1}<D_{1}<4 C_{1}$.

Now using (10)-(13), (19), (26) and (27) the expressions for $\epsilon, p_{r}, p_{t}$ and $q$ reduce the following

$$
\kappa \epsilon=\frac{\epsilon_{0}}{f^{2}}+\frac{12 \alpha(1-\sqrt{f})^{2}}{f^{3}\left[D_{2}\left(1+D_{1} r^{2}\right)\right]^{2}}
$$




$$
\begin{gathered}
\kappa p_{r}=\frac{\left(p_{r}\right)_{0}}{f^{2}}+\frac{4 \alpha^{2}(1-\sqrt{f})}{f^{\frac{5}{2}}\left[D_{2}\left(1+D_{1} r^{2}\right)\right]^{2}} \\
\kappa p_{t}=\frac{\left(p_{t}\right)_{0}}{f^{2}}+\frac{4 \alpha^{2}(1-\sqrt{f})}{f^{\frac{5}{2}}\left[D_{2}\left(1+D_{1} r^{2}\right)\right]^{2}} \\
\kappa q=\frac{8 D_{1} r\left(1+C_{1} r^{2}\right)^{2}}{D_{2} C_{2}^{2}\left(1+D_{1} r^{2}\right)^{2}} \frac{2 \alpha(1-\sqrt{f})}{f^{\frac{7}{2}}}
\end{gathered}
$$

We can see the physical parameters $\epsilon, p_{r}, p_{t}$ are finite, positive, monotonically decreasing at any instant with respect to radial coordinate for $0 \leq r \leq r_{\Sigma}$.

The fluid collapse rate $\Theta=w_{; \mu}^{\mu}$ with the help of (1), (19), (26) and (27) is

$$
\Theta=\frac{-6 \alpha(1-\sqrt{f})}{f^{\frac{3}{2}}\left[D_{2}\left(1+D_{1} r^{2}\right)\right]}
$$

where by using (18), (26) and (27) we have

$$
\alpha=\frac{2 C_{1} D_{2} r_{\Sigma}\left(1+C_{1} r_{\Sigma}^{2}\right)}{C_{2}\left(1-C_{1} r_{\Sigma}^{2}-C_{1}^{2} r_{\Sigma}^{4}\right)}
$$

The constant $\alpha$ is positive for the given range of the parameters $C_{1}, C_{2}$ and $D_{2}$.

The total energy entrapped inside the surface $\Sigma$ is given by using (6), (18), (26), (27) and (38)

$$
M(v)=\left[\frac{8 C_{1}^{2} C_{2} r_{\Sigma}^{5}(1-\sqrt{f})^{2}}{\left(1+C_{1} r_{\Sigma}^{2}\right)^{3}\left(1-C_{1} r_{\Sigma}^{2}\right)^{2}}+m_{0} f\right]_{\Sigma}
$$

where

$$
m_{0}=\frac{2 C_{1} C_{2} r_{\Sigma}^{3}}{\left(1+C_{1} r_{\Sigma}^{2}\right)^{3}}
$$

Using (4) and (27), we get the physical radius of the collapsing radiating star as

$$
R_{\Sigma}(v)=r_{\Sigma} C_{2}\left(1+C_{1} r_{\Sigma}^{2}\right)^{-1} f
$$

Using (7)-(9), (18), (26), (27) and (38) the expressions for the surface luminosity, the boundary redshift on $\Sigma$ and the luminosity for distant observer at rest at infinity respectively are

$$
\begin{gathered}
L_{\Sigma}=8\left[\frac{C_{1} r_{\Sigma}^{2}}{\left(1-C_{1}^{2} r_{\Sigma}^{4}\right)}\right]^{2} \frac{(1-\sqrt{f})}{\sqrt{f}} \\
L_{\infty}=\frac{L_{\Sigma}}{\left(1+z_{\Sigma}\right)^{2}} \\
z_{\Sigma}=\left[\frac{\sqrt{f}\left(1+C_{1} r_{\Sigma}^{2}\right)^{2}-4 C_{1} r_{\Sigma}^{2}}{\sqrt{f}\left(1-C_{1}^{2} r_{\Sigma}^{4}\right)}\right]_{\Sigma}^{-1}-1
\end{gathered}
$$


To obtain the black hole formation time, the surface redshift goes to infinity, for this the term in the parentheses of (44) goes to zero and we have

$$
\begin{gathered}
\sqrt{f_{B H}}=\frac{4 C_{1} r_{\Sigma}^{2}}{\left(1+C_{1} r_{\Sigma}^{2}\right)^{2}} \\
t_{B H}=\frac{1}{\alpha} \ln \left[\frac{1-C_{1} r_{\Sigma}^{2}}{\left(1+C_{1} r_{\Sigma}^{2}\right)}\right]^{2}
\end{gathered}
$$

\section{Temperature Profile for Collapsing Radiating Star}

To investigate the temperature inside and on the boundary surface, we utilize temperature gradient law (Israel et al. [39]; Maartens [40]; and Martinez [41])

$$
\tau\left(g^{\mu v}+w^{\mu} w^{v}\right) w^{\alpha} q_{v ; \alpha}+q^{\mu}=-\mathbb{K}\left(g^{\mu v}+w^{\mu} w^{v}\right)\left[T_{, v}+T \dot{w}_{v}\right]
$$

where $\mathbb{K}$ is the thermal conductivity and $\tau$ is the relaxation time. To get a simple estimate of the temperature evolution, we set relaxation time as zero in above expression and obtained

$$
q=-\mathbb{K} \frac{1}{B_{0}^{2} f^{2}}\left(T^{\prime}+T \frac{A_{0}^{\prime}}{A_{0}}\right)=-\frac{2 A_{0}^{\prime} \dot{f}}{A_{0}^{2} B_{0}^{2} f^{4}}
$$

The effective surface temperature observed by external observer can be calculated from the expression (Schwarzschild [42])

$$
T_{\Sigma}^{4}=\left\{\frac{1}{\pi \delta\left(r B_{0} f\right)^{2}}\right\}_{\Sigma} L_{\infty}=\frac{16 C_{1}^{2} r_{\Sigma}^{2}}{\pi \delta C_{2}^{2}\left(1-C_{1} r_{\Sigma}^{2}\right)^{2}} \frac{(1-\sqrt{f})}{f^{5 / 2}} \frac{1}{\left(1+z_{\Sigma}\right)^{2}}
$$

where for photons the constant $\delta$ is given by $\delta=\left(\pi^{2} k^{4}\right) /\left(15 \hbar^{3}\right)$, here $k$ and $\hbar$ denoting respectively Boltzmann and Plank constants. Choosing $\Omega=3$ which represents radiation interaction with matter through the diffusive approximation (Misner and Sharp [43].

The arbitrary function $T_{0}(t)$ is determined by using (48) and (49) as

$$
T_{0}(t)=\left\{\frac{16 \alpha}{3 k \gamma} \frac{\left[D_{2}\left(1+D_{1} r^{2}\right)\right]^{3}(1-\sqrt{f})}{f^{3 / 2}}\right\}_{\Sigma}+\left\{\frac{2 \alpha^{2}\left[D_{2}\left(1+D_{1} r^{2}\right)\right]^{2}}{\pi \delta r^{2}} \frac{1-\sqrt{f}}{f^{5 / 2}}\right\}_{\Sigma} \frac{1}{\left(1+z_{\Sigma}\right)^{2}}
$$

Temperature distribution throughout the interior of the collapsing radiating star is given by

$$
T^{4}=\left[\frac{T_{0}(t)}{\left[D_{2}\left(1+D_{1} r^{2}\right)\right]^{4}}-\frac{16 \alpha}{3 \gamma\left[D_{2}\left(1+D_{1} r^{2}\right)\right]} \frac{(1-\sqrt{f})}{f^{3 / 2}}\right]
$$

It follows that the surface temperature of the collapsing star tends to zero at the beginning of the collapse $[f \rightarrow 1]$ and the stage of formation of black hole $\left[z_{\Sigma} \rightarrow \infty\right]$.

\section{Conclusion}

We here presented a new radiating fluid model collapsing in the influence of its own gravity using Tewari and Charan [31] solution as seed solution. The interior fluid is spherically symmetric shear-free anisotropic and radiating away its energy in the form of radial heat flow. We have obtained a class of exact solutions by assigning different values to the parameter $n$ and proposed solutions are listed in the Table 1. Keeping in mind pressure anisotropy a simple radiating star model for $n=0$ studied in detail. Physical and thermal features of the model are significantly sound as it corresponds to well-behaved nature for the fluid density, both the radial 
Table 1. Parametric class of solutions.

\begin{tabular}{|c|c|c|c|c|c|}
\hline S.N. & $n$ & l & $A_{0}$ & $B_{0}$ & $\delta(r)$ \\
\hline 1 & $\frac{5 \pm \sqrt{41}}{2}$ & 2 & $D_{2}\left(1+D_{1} r^{2}\right)\left(1+C_{1} r^{2}\right)^{\frac{5 \pm \sqrt{41}}{6}}$ & $C_{2}\left(1+C_{1} r^{2}\right)^{\frac{1}{3}}$ & $\left(\frac{3 \pm \sqrt{41}}{3}\right) \frac{4 D_{1} C_{1} r^{2}}{\left(1+D_{1} r^{2}\right)\left(1+C_{1} r^{2}\right)}$ \\
\hline 2 & $2 \pm \sqrt{7}$ & 1 & $D_{2}\left(1+D_{1} r^{2}\right)\left(1+C_{1} r^{2}\right)^{\frac{2 \pm \sqrt{7}}{2}}$ & $C_{2}\left(1+C_{1} r^{2}\right)^{\frac{1}{2}}$ & $(1 \pm \sqrt{7}) \frac{4 D_{1} C_{1} r^{2}}{\left(1+D_{1} r^{2}\right)\left(1+C_{1} r^{2}\right)}$ \\
\hline 3 & $\frac{3 \pm \sqrt{17}}{2}$ & 0 & $D_{2}\left(1+D_{1} r^{2}\right)\left(1+C_{1} r^{2}\right)^{\frac{3 \pm \sqrt{17}}{2}}$ & $C_{2}\left(1+C_{1} r^{2}\right)$ & $(1 \pm \sqrt{17}) \frac{4 D_{1} C_{1} r^{2}}{\left(1+D_{1} r^{2}\right)\left(1+C_{1} r^{2}\right)}$ \\
\hline 4 & $-1+\sqrt{2}$ & $-5+2 \sqrt{2}$ & $D_{2}\left(1+D_{1} r^{2}\right)\left(1+C_{1} r^{2}\right)^{\frac{-\sqrt{2}}{4}}$ & $C_{2}\left(1+C_{1} r^{2}\right)^{\frac{-(2+\sqrt{2})}{4}}$ & $\frac{4 D_{1} C_{1} r^{2}}{\left(1+D_{1} r^{2}\right)\left(1+C_{1} r^{2}\right)}$ \\
\hline 5 & 0 & -2 & $D_{2}\left(1+D_{1} r^{2}\right)$ & $C_{2}\left(1+C_{1} r^{2}\right)^{-1}$ & $\frac{8 D_{1} C_{1} r^{2}}{\left(1+D_{1} r^{2}\right)\left(1+C_{1} r^{2}\right)}$ \\
\hline 6 & 1 & -2 & $D_{2}\left(1+D_{1} r^{2}\right)\left(1+C_{1} r^{2}\right)^{-1}$ & $C_{2}\left(1+C_{1} r^{2}\right)^{-1}$ & 0 \\
\hline 7 & $-1-\sqrt{2}$ & $-5-2 \sqrt{2}$ & $D_{2}\left(1+D_{1} r^{2}\right)\left(1+C_{1} r^{2}\right)^{\frac{\sqrt{2}}{4}}$ & $C_{2}\left(1+C_{1} r^{2}\right)^{\frac{-(2+\sqrt{2})}{4}}$ & $\frac{4 D_{1} C_{1} r^{2}}{\left(1+D_{1} r^{2}\right)\left(1+C_{1} r^{2}\right)}$ \\
\hline 8 & -2 & -8 & $D_{2}\left(1+D_{1} r^{2}\right)\left(1+C_{1} r^{2}\right)^{\frac{2}{7}}$ & $C_{2}\left(1+C_{1} r^{2}\right)^{\frac{-1}{7}}$ & $\frac{24 D_{1} C_{1} r^{2}}{7\left(1+D_{1} r^{2}\right)\left(1+C_{1} r^{2}\right)}$ \\
\hline 9 & -3 & -8 & $D_{2}\left(1+D_{1} r^{2}\right)\left(1+C_{1} r^{2}\right)^{\frac{3}{7}}$ & $C_{2}\left(1+C_{1} r^{2}\right)^{\frac{-1}{7}}$ & $\frac{32 D_{1} C_{1} r^{2}}{7\left(1+D_{1} r^{2}\right)\left(1+C_{1} r^{2}\right)}$ \\
\hline 10 & -4 & $-\frac{26}{3}$ & $D_{2}\left(1+D_{1} r^{2}\right)\left(1+C_{1} r^{2}\right)^{\frac{12}{23}}$ & $C_{2}\left(1+C_{1} r^{2}\right)^{\frac{-3}{23}}$ & $\frac{120 D_{1} C_{1} r^{2}}{23\left(1+D_{1} r^{2}\right)\left(1+C_{1} r^{2}\right)}$ \\
\hline 11 & $-\frac{5}{3}$ & $-\frac{26}{3}$ & $D_{2}\left(1+D_{1} r^{2}\right)\left(1+C_{1} r^{2}\right)^{\frac{5}{23}}$ & $C_{2}\left(1+C_{1} r^{2}\right)^{\frac{-3}{23}}$ & $\frac{64 D_{1} C_{1} r^{2}}{23\left(1+D_{1} r^{2}\right)\left(1+C_{1} r^{2}\right)}$ \\
\hline 12 & -5 & $-\frac{19}{2}$ & $D_{2}\left(1+D_{1} r^{2}\right)\left(1+C_{1} r^{2}\right)^{\frac{10}{17}}$ & $C_{2}\left(1+C_{1} r^{2}\right)^{\frac{-2}{17}}$ & $\frac{96 D_{1} C_{1} r^{2}}{17\left(1+D_{1} r^{2}\right)\left(1+C_{1} r^{2}\right)}$ \\
\hline 13 & $-\frac{3}{2}$ & $-\frac{19}{2}$ & $D_{2}\left(1+D_{1} r^{2}\right)\left(1+C_{1} r^{2}\right)^{\frac{3}{17}}$ & $C_{2}\left(1+C_{1} r^{2}\right)^{\frac{-2}{17}}$ & $\frac{40 D_{1} C_{1} r^{2}}{17\left(1+D_{1} r^{2}\right)\left(1+C_{1} r^{2}\right)}$ \\
\hline 14 & -6 & $-\frac{52}{5}$ & $D_{2}\left(1+D_{1} r^{2}\right)\left(1+C_{1} r^{2}\right)^{\frac{30}{47}}$ & $C_{2}\left(1+C_{1} r^{2}\right)^{\frac{-5}{47}}$ & $\frac{280 D_{1} C_{1} r^{2}}{47\left(1+D_{1} r^{2}\right)\left(1+C_{1} r^{2}\right)}$ \\
\hline 15 & $-\frac{7}{5}$ & $-\frac{52}{5}$ & $D_{2}\left(1+D_{1} r^{2}\right)\left(1+C_{1} r^{2}\right)^{\frac{7}{47}}$ & $C_{2}\left(1+C_{1} r^{2}\right)^{\frac{-5}{47}}$ & $\frac{96 D_{1} C_{1} r^{2}}{47\left(1+D_{1} r^{2}\right)\left(1+C_{1} r^{2}\right)}$ \\
\hline 16 & -7 & $-\frac{34}{3}$ & $D_{2}\left(1+D_{1} r^{2}\right)\left(1+C_{1} r^{2}\right)^{\frac{21}{31}}$ & $C_{2}\left(1+C_{1} r^{2}\right)^{\frac{-3}{31}}$ & $\frac{192 D_{1} C_{1} r^{2}}{31\left(1+D_{1} r^{2}\right)\left(1+C_{1} r^{2}\right)}$ \\
\hline 17 & $-\frac{4}{3}$ & $-\frac{34}{3}$ & $D_{2}\left(1+D_{1} r^{2}\right)\left(1+C_{1} r^{2}\right)^{\frac{4}{31}}$ & $C_{2}\left(1+C_{1} r^{2}\right)^{\frac{-3}{31}}$ & $\frac{56 D_{1} C_{1} r^{2}}{31\left(1+D_{1} r^{2}\right)\left(1+C_{1} r^{2}\right)}$ \\
\hline 18 & -8 & $-\frac{86}{7}$ & $D_{2}\left(1+D_{1} r^{2}\right)\left(1+C_{1} r^{2}\right)^{\frac{56}{79}}$ & $C_{2}\left(1+C_{1} r^{2}\right)^{\frac{-7}{79}}$ & $\frac{504 D_{1} C_{1} r^{2}}{79\left(1+D_{1} r^{2}\right)\left(1+C_{1} r^{2}\right)}$ \\
\hline 19 & $-\frac{9}{7}$ & $-\frac{86}{7}$ & $D_{2}\left(1+D_{1} r^{2}\right)\left(1+C_{1} r^{2}\right)^{\frac{9}{79}}$ & $C_{2}\left(1+C_{1} r^{2}\right)^{\frac{-7}{79}}$ & $\frac{128 D_{1} C_{1} r^{2}}{79\left(1+D_{1} r^{2}\right)\left(1+C_{1} r^{2}\right)}$ \\
\hline
\end{tabular}




\section{Continued}

\begin{tabular}{|c|c|c|c|c|c|}
\hline 20 & -9 & $-\frac{53}{4}$ & $D_{2}\left(1+D_{1} r^{2}\right)\left(1+C_{1} r^{2}\right)^{\frac{36}{49}}$ & $C_{2}\left(1+C_{1} r^{2}\right)^{\frac{-4}{49}}$ & $\frac{320 D_{1} C_{1} r^{2}}{49\left(1+D_{1} r^{2}\right)\left(1+C_{1} r^{2}\right)}$ \\
\hline \multirow{2}{*}{21} & 5 & 53 & \multirow{2}{*}{$D_{2}\left(1+D_{1} r^{2}\right)\left(1+C_{1} r^{2}\right)^{\frac{5}{99}}$} & \multirow{2}{*}{$C_{2}\left(1+C_{1} r^{2}\right)^{\frac{-4}{49}}$} & $72 D_{1} C_{1} r^{2}$ \\
\hline & $-\overline{4}$ & 4 & & & $\overline{49\left(1+D_{1} r^{2}\right)\left(1+C_{1} r^{2}\right)}$ \\
\hline \multirow{2}{*}{22} & 10 & 128 & \multirow{2}{*}{$D_{2}\left(1+D_{1} r^{2}\right)\left(1+C_{1} r^{2}\right)^{\frac{90}{119}}$} & \multirow{2}{*}{$C_{2}\left(1+C_{1} r^{2}\right)^{\frac{-9}{119}}$} & $792 D_{1} C_{1} r^{2}$ \\
\hline & -10 & $\overline{9}$ & & & $119\left(1+D_{1} r^{2}\right)\left(1+C_{1} r^{2}\right)$ \\
\hline \multirow{2}{*}{23} & 11 & 128 & \multirow{2}{*}{$D_{2}\left(1+D_{1} r^{2}\right)\left(1+C_{1} r^{2}\right)^{\frac{11}{119}}$} & \multirow{2}{*}{$C_{2}\left(1+C_{1} r^{2}\right)^{\frac{-9}{119}}$} & $160 D_{1} C_{1} r^{2}$ \\
\hline & $-\overline{9}$ & $\overline{9}$ & & & $119\left(1+D_{1} r^{2}\right)\left(1+C_{1} r^{2}\right)$ \\
\hline \multirow{2}{*}{24} & & 76 & \multirow{2}{*}{$D_{2}\left(1+D_{1} r^{2}\right)\left(1+C_{1} r^{2}\right)^{\frac{55}{71}}$} & \multirow{2}{*}{$C_{2}\left(1+C_{1} r^{2}\right)^{\frac{-5}{71}}$} & $480 D_{1} C_{1} r^{2}$ \\
\hline & -11 & 5 & & & $\overline{71\left(1+D_{1} r^{2}\right)\left(1+C_{1} r^{2}\right)}$ \\
\hline \multirow{2}{*}{25} & 6 & 76 & \multirow{2}{*}{$D_{2}\left(1+D_{1} r^{2}\right)\left(1+C_{1} r^{2}\right)^{\frac{6}{71}}$} & \multirow{2}{*}{$C_{2}\left(1+C_{1} r^{2}\right)^{\frac{-5}{71}}$} & $88 D_{1} C_{1} r^{2}$ \\
\hline & 5 & $\overline{5}$ & & & $\overline{71\left(1+D_{1} r^{2}\right)\left(1+C_{1} r^{2}\right)}$ \\
\hline
\end{tabular}

and tangential pressures and the flux density throughout the fluid sphere. We observed that the function $f(t)$ decreases monotonically from the value $f(t)=1$ at $t=-\infty$ to $f(t)=0$ at $t=0$. The apparent luminosity as observed by the distant observer at rest at infinity is zero in remote past at the instance when the collapse begins and at the stage of black hole formation.

Particularly we have constructed a radiating star model by taking suitable parameters $C_{1}=0.01, C_{2}=4$, $D_{2}=0.5, D_{1}=3 C_{1}$. Initially when $f(t) \rightarrow 1$ both the mass and physical radius of a core of pre Supernovae were $5 M_{\Theta}$ and $15.8 \mathrm{~km}$ and they remain $2.6 M_{\Theta}$ and $6.30 \mathrm{~km}$ respectively at the time of black hole formation. The time of black hole formation is observed as $0.62996 \mathrm{~S}$. We observed that the model is well behaved for the chosen constraints and there are a number of such sets for which the solution is well behaved. Thus the parametric class of solution and constructed table is very fruitful for further study of radiating and static compact star's modeling. The surface temperature of the collapsing radiating star tends to zero at the beginning of the collapse $[f \rightarrow 1]$ and the stage of formation of black hole $\left[z_{\Sigma} \rightarrow \infty\right]$.

\section{Acknowledgements}

Authors express their gratitude and thanks to the anonymous referee for his rigorous review and valuable suggestions.

\section{References}

[1] Joshi, P.S. and Malafarina, D. (2011) Recent Developments in Gravitational Collapse and Spacetime Singularities. International Journal of Modern Physics D, 20, 2641-2729. http://dx.doi.org/10.1142/S0218271811020792

[2] Penrose, R. (1969) Gravitational Collapse: The Role of General Relativity. La Rivista del Nuovo Cimento, 1, $252-276$.

[3] Virbhadra, K.S. (2009) Relativistic Images of Schwarzschild Black Hole Lensing. Physical Review D, 79, 083004. http://dx.doi.org/10.1103/PhysRevD.79.083004

[4] Oppenheimer, J.R. and Snyder, H. (1939) Continued Gravitational Contraction. Physical Review, 56, 455. http://dx.doi.org/10.1103/PhysRev.56.455

[5] Vaidya, P.C. (1951) The Gravitational Field of a Radiating Star. Proceedings of the National Academy of Sciences, India, Section A: Physical Sciences, 33, 264-276.

[6] Vaidya, P.C. (1953) Newtonian Time in General Relativity. Nature, 171, 260-261. http://dx.doi.org/10.1038/171260a0

[7] Santos, N.O. (1985) Non-Adiabatic Radiating Collapse. Monthly Notices of the Royal Astronomical Society, 216, 403410. http://dx.doi.org/10.1093/mnras/216.2.403

[8] Herrera, L. and Santos, N.O. (2004) Dynamics of Dissipative Gravitational Collapse. Physical Review D, 70, 084004. http://dx.doi.org/10.1103/PhysRevD.70.084004 
[9] Mitra, A. (2006) Why Gravitational Contraction Must Be Accompanied by Emission of Radiation Both in Newtonian and Einstein Gravity. Physical Review D, 74, 024010. http://dx.doi.org/10.1103/PhysRevD.74.024010

[10] Tewari, B.C. (1988) Radiating Fluid Spheres in General Relativity. Astrophysics and Space Science, 149, $233-239$. http://dx.doi.org/10.1007/BF00639793

[11] Tewari, B.C. (1994) Relativistic Radiating Fluid Distribution. Indian Journal of Pure and Applied Physics, 32, 504.

[12] Tewari, B.C. (2006) Relativistic Model for Radiating Star. Astrophysics and Space Science, 306, 273-277. http://dx.doi.org/10.1007/s10509-006-9273-y

[13] Tewari, B.C. (2010) Radiating Fluid Balls in General Relativity. VDM Verlag, Saarbrucken.

[14] de Oliveira, A.K.G., Santos, N.O. and Kolassis, C.A. (1985) Collapse of a Radiating Star. Monthly Notices of the Royal Astronomical Society, 216, 1001-1011. http://dx.doi.org/10.1093/mnras/216.4.1001

[15] Bonnor W. B., de Oliveira, A.K.G. and Santos, N.O. (1989) Radiating Spherical Collapse. Physical Review Letters, 181, 269-326. http://dx.doi.org/10.1016/0370-1573(89)90069-0

[16] Herrera, L., Di Prisco, A., Ospino, J., Fuenmayor, E. and Triconis, O. (2009) Structure and Evolution of Self-Gravitating Objects and the Orthogonal Splitting of the Riemann Tensor. Physical Review D, 79, Article ID: 064025. http://dx.doi.org/10.1103/PhysRevD.79.064025

[17] Maharaj, S.D. and Govender, M. (2005) Radiating Collapse with Vanishing Weyl Stresses. International Journal of Modern Physics D, 14, 667-676. http://dx.doi.org/10.1142/S0218271805006584

[18] Ivanov, B.V. (2012) Collapsing Shear-Free Perfect Fluid Spheres with Heat Flow. General Relativity and Gravitation, 44, 1835-1855. http://dx.doi.org/10.1007/s10714-012-1370-3

[19] Tewari, B.C. (2012) Relativistic Collapsing Radiating Stars. Astrophysics and Space Science, 342, 73-77. http://dx.doi.org/10.1007/s10509-012-1141-3

[20] Tewari, B.C. (2013) Collapsing Shear-Free Radiating Fluid Spheres. General Relativity and Gravitation, 45, 15471558. http://dx.doi.org/10.1007/s10714-013-1545-6

[21] Pinheiro, G. and Chan, R. (2013) Radiating Shear-Free Gravitational Collapse with Charge. General Relativity and Gravitation, 45, 243-261. http://dx.doi.org/10.1007/s10714-012-1468-7

[22] Tewari, B.C. and Charan, K. (2014) Radiating Star, Shear-Free Gravitational Collapse without Horizon. Astrophysics and Space Science, 351, 613-617. http://dx.doi.org/10.1007/s10509-014-1851-9

[23] Tewari, B.C. and Charan, K. (2015) Dissipative Spherical Gravitational Collapse of Isotropic Fluid. Journal of Modern Physics, 6, 453-462. http://dx.doi.org/10.4236/jmp.2015.64049

[24] Sharif, M. and Iftikhar, S. (2015) Charged Dissipative Collapse of Shearing Viscous Star. Astrophysics and Space Science, 357, 79. http://dx.doi.org/10.1007/s10509-015-2246-2

[25] Bowers, R.L. and Liang, E.P.T. (1974) Anisotropic Spheres in General Relativity. Astrophysical Journal, 188, $657-665$. http://dx.doi.org/10.1086/152760

[26] Herrera, L. and Santos, N.O. (1997) Local Anisotropy in Self-Gravitating Systems. Physics Reports, 286, 53-130. http://dx.doi.org/10.1016/S0370-1573(96)00042-7

[27] Herrera, L., Di Prisco, A., Hernandez-Pastoraand, J.L. and Santos, N.O. (1998) On the Role of Density Inhomogeneity and Local Anisotropy in the Fate of Spherical Collapse. Physics Letters A, 237, 113-118. http://dx.doi.org/10.1016/S0375-9601(97)00874-8

[28] Ivanov, B.V. (2010) Evolving Spheres of Shear-Free Anisotropic Fluid. International Journal of Modern Physics A, 25, 3975-3991. http://dx.doi.org/10.1142/S0217751X10050202

[29] Ivanov, B.V. (2011) Self-Gravitating Spheres of Anisotropic Fluid in Geodesic Flow. International Journal of Modern Physics D, 20, 319-334. http://dx.doi.org/10.1142/S0218271811018858

[30] Reddy, K.P., Govender, M. and Maharaj, S.D. (2015) Impact of Anisotropic Stresses during Dissipative Gravitational Collapse. General Relativity and Gravitation, 47, 35. http://dx.doi.org/10.1007/s10714-015-1880-x

[31] Tewari, B.C. and Charan, K. (2015) Horizon Free Eternally Collapsing Anisotropic Radiating Star. Astrophysics and Space Science, 357, 107. http://dx.doi.org/10.1007/s10509-015-2335-2

[32] Tewari, B.C. and Charan, K. (2015) Gravitational Collapse, Shear-Free Anisotropic Radiating Star. arxiv:1503.02165

[33] Govender, M., Bogadi, R.S., Lortan, D.B. and Maharaj, S.D. (2016) Radiating Collapse in the Presence of Anisotropic Stresses. International Journal of Modern Physics D, 25, Article ID: 1650037. http://dx.doi.org/10.1142/S0218271816500371

[34] Ivanov, B.V. (2016) All Solutions for Geodesic Anisotropic Soherical Collapse with Shear and Heat Flow. Astrophysics and Space Science, 361, 18. http://dx.doi.org/10.1007/s10509-015-2603-1 
[35] Ivanov, B.V. (2016) A Different Approach to Anisotropic Spherical Collapse with Shear and Heat Radiation. International Journal of Modern Physics D, 25, Article ID: 1650049. http://dx.doi.org/10.1142/S0218271816500498

[36] Cahill, M.E. and McVittie, G.C. (1970) Spherical Symmetry and Mass Energy in General Relativity I. General Theory. Journal of Mathematical Physics, 11, 1382-1391. http://dx.doi.org/10.1063/1.1665273

[37] Misner, C.W. and Sharp, D.H. (1964) Relativistic Equations for Adiabatic Spherically Symmetric Gravitational Collapse. Physical Review B, 136, 571-576. http://dx.doi.org/10.1103/PhysRev.136.B571

[38] Banerjee, A., Chatterjee, S. and Dadhich, N. (2002) Spherical Collapse with Heat Flow and without Horizon. Modern Physics Letters A, 17, 2335-2339. http://dx.doi.org/10.1142/S0217732302008320

[39] Israel, W. and Stewart, J. (1979) Transient Relativistic Thermodynamics and Kinetic Theory. Annals of Physics, 118, 341-372. http://dx.doi.org/10.1016/0003-4916(79)90130-1

[40] Maartens, R. (1995) Dissipative Cosmology. Classical and Quantum Gravity, 12, 1455-1465. http://dx.doi.org/10.1088/0264-9381/12/6/011

[41] Martinez, J. (1996) Transport Processes in the Gravitational Collapse of an Anisotropic Fluid. Physical Review D, 53, 6921-6940. http://dx.doi.org/10.1103/PhysRevD.53.6921

[42] Schwarzschild, M. (1958) Structure and Evolution of stars. Dover, New York.

[43] Misner, C.W. and Sharp, D.H. (1965) Spherical Gravitational Collapse with Energy Transport by Radiative Diffusion. Physics Letters, 15, 279-281. http://dx.doi.org/10.1016/0031-9163(65)91247-3 